\title{
Exploration and Inventory of Native Orchid Germplasm in West Borneo, Indonesia
}

\author{
Chairani Siregar ${ }^{1}$ \\ Department of Horticulture, College of Agriculture, University of \\ Tanjungpura, Jln. Ahmad Yani Pontianak 78124, Kalimantan Barat, \\ Indonesia
}

Additional index words. orchid species, germplasm

\begin{abstract}
Borneo (Kalimantan) is the third largest island in the world. It is rich with various indigenous orchid species that grow epiphytically, terrestrially, or saprophytically in the forests. Its rain forests are also home to some rare species such as some Aërides sp., Bulbophyllum sp., Cymbidium sp., Dendrobium sp., Dimorphorchis sp., Grammatophyllum sp., Paphiopedilum sp., Phalaenopsis sp., Paraphalaenopsis sp., and Vanda sp., all of which have a very high economic value. These species are endangered and some of them may have not yet been found or discovered, because of the loss of habitat resulting from fire, forest damage, illegal logging, and orchid hunting either by domestic or foreign collectors. Until recently, there are only a few records on the orchid native to West Borneo. For this reason, a research was conducted to identify and create an inventory of all orchid species that exist in West Borneo before they become extinct along with their habitat and to conserve them ex situ. This research was conducted in 10 counties and one municipal city in West Borneo, and inventory was done through exploration. Orchids found were recorded and identified into their genera and their species by visual examination of vegetative and floral characteristics, respectively. A total of 197 species of orchids from 66 genera were identified, and among those, 27 species live as terrestrials, 169 species live as epiphytes, and one species lives as both an epiphyte and terrestrial.
\end{abstract}

Orchids are members of the Orchidaceae, the largest family among flowering plants, which includes 25,000 species from 850 genera. It is estimated that 2500 to 3000 orchid species grow in the forests of Borneo (Irawati, 2002).

In their natural habitats, most orchids live as epiphytes in the forest trees. Increasing forest exploitation, either legally or illegally, and excessive logging have caused damage to the forests of West Borneo. Gold mining, wild forest fires, and illegal burning to establish a new agriculture land occur often and are some of the reasons orchids are becoming extinct. There are also economic factors contributing to the endangerment of Borneo's orchids such as illegal collecting and selling of wild orchids by collectors (orchid lovers) and the increasing demand for orchids by neighboring countries such as Malaysia and Brunei Darussalam. These conditions point to the urgency of the need to conserve native orchids in West Borneo.

Conservancy efforts should be conducted in accordance with the following Indonesian legislations: UU No. 5 ratified in 1990, The Conservation of Biological Natural Resources and Its Ecosystem (Indonesian Ministry

Received for publication 9 July 2007. Accepted for publication 10 Oct. 2007.

I thank Deliana Siregar McCreary and Rieza Soelaeman for reviewing and editing this paper.

${ }^{1}$ To whom reprint requests should be addressed; e-mail chairani8@yahoo.com of the Environment, 1990); UU No. 5 ratified in 1994, The Conservation of Biological Diversity (Indonesian Ministry of the Environment, 1994); and PP No. 7 ratified in 1999, The Conservation of Flora and fauna (Indonesian Ministry of the Environment, 1999).

According to Global Forest Watch (2002), Indonesia is one of the countries experiencing the most drastic loss of forestland in the world. It was estimated in 1996 that $\approx 1$ million ha of Indonesian forestland was being destroyed every year, including $\approx 1.7$ million ha every year during the 1990 s such that it was estimated that the forests in Borneo will have completely vanished by 2010. It is as a result of these figures that the author tries to promote conservation efforts, which include conserving native orchids by ex situ processes and making an inventory and identifying native orchids in West Borneo in accordance with the legislation mentioned previously.

\section{Materials and Methods}

Time and location. The research was conducted in 3 years, from 2002 to 2005 . The author explored forests in all 10 counties and one municipal city in West Borneo. The counties were Sambas, Bengkayang, Pontianak, Sekadau, Sanggau, Kapuas Hulu, Sintang, Landak, Malawi, and Ketapang and the municipal city was Pontianak City.

Materials and tools. Materials used for this research were orchid plant guidebooks.
Several reference books written by Banks (1999, 2004a, 2004b), Chan et al. (1994), Clayton (2002), Cribb (1997), Dixon et al. (2003), Dressler (1993), Marinelli (2004), Moeso (1988), O’Byrne (2001), Teo (1995), Vermeulen (1991), and Wood (1997, 2001, 2003) were used for orchid determination. Especially useful were Orchids of Borneo Vol. 1 (Chan et al., 1994), Orchids of Borneo Vol. 2 (Vermeulen, 1991), Orchids of Borneo Vol. 3 (Wood, 1997), Orchids of Borneo Vol. 4 (Wood, 2003), and $A$ to $Z$ of South East Asian Orchid Species (O'Byrne, 2001).

The tools used were a camera, a compass, measuring tapes, clippers, wrapping paper, plastic sacks, paper bags, ropes, and a forest map of West Borneo.

Research methods. Explorative inventory through purposive sampling was conducted to identify the native orchids into their genera and species. Purposive sampling involved choosing exploration sites based on the existence of forests, both primary and secondary, and along riverbanks within counties. Epiphytic orchids were collected from the forest by climbing the trees or from the dead plant, and terrestrial ones were dug, including the soil, surrounding the roots. They were put into paper bags, and their habitats and their locations were recorded. Then, they were taken to the nursery and grown ex situ. Fresh flowers and their pictures were also taken for identification. Genus and species identifications were determined by visual examination of vegetative and floral characteristics, respectively. Identification was accomplished using the reference guidebooks mentioned previously. The average temperature in the forests of West Borneo is 24 to $33^{\circ} \mathrm{C}$, relative humidity $60 \%$ to $80 \%$, light intensity is 7000 to $80001 \mathrm{x}$, soil types are organic soil (peat) and mineral soils (yellowish red podzolic and alluvial), and $\mathrm{pH}$ of the soil is 4.0 to 5.5 (soil information needed for terrestrial orchids only). This information was recorded to grow the orchids ex situ.

\section{Results and Discussion}

One hundred ninety-seven orchid species from 66 genera have been identified, including some with high economic value and others with low economic value. Among these orchids, 27 species live as terrestrials, 169 species live as epiphytes in the forest, and one species lives as both an epiphyte and terrestrial (Table 1). In addition, the identification of 200 taxa is still pending, because these plants have not flowered at the time this article was written. The author found that orchid species were not uniformly distributed in West Borneo. Some species have become vulnerable, some have become endangered, whereas others have become almost extinct. Some vulnerable species such as Aërides odorata is still abundant, especially in Sambas, Landak, and Bengkayang counties. Similarly, Arundina graminifolia and Bromheadia finlaysoniana are easily found in their habitats in many counties. 


\begin{tabular}{|c|c|c|}
\hline No. & Orchid name & $\begin{array}{c}\text { Growth } \\
\text { classification }\end{array}$ \\
\hline 1 & Acanthepipium eburneum Kraenzl. & Terrestrial \\
\hline 2 & $\begin{array}{l}\text { Acanthepipium lilacinum } \\
\text { J.J. Wood \& C.L. Chan }\end{array}$ & Terrestrial \\
\hline 3 & Acriopsis densiflora Lindley & Epiphyte \\
\hline 4 & Acriopsis liliifolia (Koen.) Ormerod & Epiphyte \\
\hline 5 & Adenoncos parviflora Ridl. & Epiphyte \\
\hline 6 & Aërides odorata Lour. & Epiphyte \\
\hline 7 & Agrostophyllum laxum J.J. Sm. & Epiphyte \\
\hline 8 & Anoectochilus albolineatus Par. et Rchb. f. & Terrestrial \\
\hline 9 & Appendicula anceps $\mathrm{Bl}$. & Epiphyte \\
\hline 10 & Appendicula elegans $\mathrm{Rchb} . \mathrm{f}$. & Epiphyte \\
\hline 11 & Appendicula torta $\mathrm{B} 1$. & Epiphyte \\
\hline 12 & Appendicula undulata $\mathrm{B} 1$. & Epiphyte \\
\hline 13 & Arachnis breviscapa (J.J. Sm.) J.J. Sm. & Epiphyte \\
\hline 14 & Arachnis flosaeris (Lind.) Rchb. f. & Epiphyte \\
\hline 15 & Arachnis hookeriana (Rchb.f.) Rchb. f. & Epiphyte \\
\hline 16 & Arundina graminifolia (D. Don.) Hochr. & Terrestrial \\
\hline 17 & Brachypeza indusiata (Rchb. f.) Garay & Epiphyte \\
\hline 18 & Brachypeza zamboangensis (Ames) Garay & Epiphyte \\
\hline 19 & Bromheadia finlaysoniana (Lind.) Rchb. f. & Terrestrial \\
\hline 20 & Bulbophyllum acuminatum (Ridl.) Ridl. & Epiphyte \\
\hline 21 & Bulbophyllum anceps J.J. Sm. & Epiphyte \\
\hline 22 & Bulbophyllum auratum (Lindl.) Rchb. f. & Epiphyte \\
\hline 23 & Bulbophyllum beccarii Rchb. f. & Epiphyte \\
\hline 24 & Bulbophyllum blumei (Lindl.) J.J. Sm. & Epiphyte \\
\hline 25 & Bulbophyllum brienianum (Rolfe) Ames & Epiphyte \\
\hline 26 & Bulbophyllum cirlihanensis Schltr. & Epiphyte \\
\hline 27 & Bulbophyllum connatum Carr. & Epiphyte \\
\hline 28 & Bulbophyllum dearei (Hort.) Rchb. f. & Epiphyte \\
\hline 29 & Bulbophyllum epicrianthes Hook. f. & Epiphyte \\
\hline 30 & Bulbophyllum flavescens (B1.) Lindl. & Epiphyte \\
\hline 31 & Bulbophyllum gracillimum (Rolfe) Rolfe & Epiphyte \\
\hline 32 & Bulbophyllum lepidum (B1.) J.J. Sm. & Epiphyte \\
\hline 33 & Bulbophyllum lobbii Lindl. & Epiphyte \\
\hline 34 & Bulbophyllum macranthum Lindl. & Epiphyte \\
\hline 35 & Bulbophyllum macrochillum Rolfe. & Epiphyte \\
\hline 36 & Bulbophyllum medusae (Lindl.) Rchb. f. & Epiphyte \\
\hline 37 & Bulbophyllum mirum J.J. Sm. & Epiphyte \\
\hline 38 & Bulbophyllum mutabile (B1.) Lindl. & Epiphyte \\
\hline 39 & Bulbophyllum patens King ex Hk. f. & Epiphyte \\
\hline 40 & Bulbophyllum pileatum Schltr. & Epiphyte \\
\hline 41 & Bulbophyllum purpurascens T. \& B. & Epiphyte \\
\hline 42 & Bulbophyllum refractilingue J.J. Sm. & Epiphyte \\
\hline 43 & Bulbophyllum reticulatum Batem ex Hook. $\mathrm{f}$. & Epiphyte \\
\hline 44 & Bulbophyllum uniflorum Hook. & Epiphyte \\
\hline 45 & Bulbophyllum vaginatum (Lidl.) Rchb. f. & Epiphyte \\
\hline 46 & Bulbophyllum vanvuurenii J.J. Sm. & Epiphyte \\
\hline 47 & Calanthe vestita Lindl. & Terrestrial \\
\hline 48 & Chelonistele sulphurea (B1.) Pfitz. & Epiphyte \\
\hline 49 & Chroniochilus virescens (Ridl.) Holltum & Epiphyte \\
\hline 50 & Claderia viridiflora Hook. f. & Terrestrial \\
\hline 51 & Cleisostoma scortechinii (Hook. f.) Garay & Epiphyte \\
\hline 52 & Cleisostoma simondii (Gagnep.) Seidenf. & Epiphyte \\
\hline 53 & Cleisostoma subulatus Rchb. f. & Epiphyte \\
\hline 54 & Coelogyne asperata Lindl. & Epiphyte \\
\hline 55 & Coelogyne eberhardtii Gagnep. & Epiphyte \\
\hline 56 & Coelogyne foerstermannii Rchbf. & Epiphyte \\
\hline 57 & Coelogyne mayeriana $\mathrm{Rchbf}$. & Epiphyte \\
\hline 58 & Coelogyne pandurata Lindl. & Epiphyte \\
\hline 59 & Coelogyne rochussenii De Vr. & Epiphyte \\
\hline 60 & Coelogyne rumphii Rchb. f. & Epiphyte \\
\hline 61 & Coelogyne speciosa Lindl. & Epiphyte \\
\hline 62 & Coelogyne squamulosa J.J. Sm. & Epiphyte \\
\hline 63 & Coelogyne swaniana Rolfe & Epiphyte \\
\hline 64 & Coelogyne verrucosa S.E.C. Sierra & Epiphyte \\
\hline 65 & Cordiglottis filiformis (Hk. f.) Garay & Epiphyte \\
\hline 66 & Cymbidium bicolor Lindl. & Epiphyte \\
\hline 67 & Cymbidium finlaysonianum Lindl. & Epiphyte \\
\hline 68 & Dendrobium acerosum Lindl. & Epiphyte \\
\hline 69 & Dendrobium anosmum Lindl. & Epiphyte \\
\hline 70 & Dendrobium babiense J.J. Sm. & Epiphyte \\
\hline 71 & Dendrobium cinerium J.J. Sm. & Epiphyte \\
\hline 72 & Dendrobium compressistyllum J.J. Sm. & Epiphyte \\
\hline
\end{tabular}

\begin{tabular}{|c|c|c|}
\hline No. & Orchid name & $\begin{array}{c}\text { Growth } \\
\text { classification }\end{array}$ \\
\hline 73 & Dendrobium crumenatum Sw. & Epiphyte \\
\hline 74 & Dendrobium distichum Rchb. f. & Epiphyte \\
\hline 75 & Dendrobium endertii J.J. Sm. & Epiphyte \\
\hline 76 & Dendrobium grande Hook. f. & Epiphyte \\
\hline 77 & Dendrobium hallieri J.J. Sm. & Epiphyte \\
\hline 78 & Dendrobium hepaticum J.J. Sm. & Epiphyte \\
\hline 79 & Dendrobium lamellatum B1. & Epiphyte \\
\hline 80 & Dendrobium leonis (Lindl.) Rchb. f. & Epiphyte \\
\hline 81 & Dendrobium linearifolium Hook. f. & Epiphyte \\
\hline 82 & Dendrobium macrophyllum A. Rich. & Epiphyte \\
\hline 83 & Dendrobium microglaphys Rchbf. & Epiphyte \\
\hline 84 & Dendrobium moquetteanum J.J. Sm. & Epiphyte \\
\hline 85 & $\begin{array}{l}\text { Dendrobium oblongum } \\
\text { Ames \& C. Schweinf }\end{array}$ & Epiphyte \\
\hline 86 & Dendrobium pachyphyllum (Kuntz) Bakh. f. & Epiphyte \\
\hline 87 & Dendrobium pandaneti Ridl. & Epiphyte \\
\hline 88 & $\begin{array}{l}\text { Dendrobium patentilobum } \\
\text { Ames \& C. Schweinf }\end{array}$ & Epiphyte \\
\hline 89 & Dendrobium sanguinolentum Lindl. & Epiphyte \\
\hline 90 & Dendrobium secundum (B1.) Lindl. & Epiphyte \\
\hline 91 & Dendrobium singaporense Hawkes \& Heller & Epiphyte \\
\hline 92 & Dendrobium singkawangense J.J. Sm. & Epiphyte \\
\hline 93 & Dendrobium smithianum Schltr. & Epiphyte \\
\hline 94 & Dendrobium spurium (B1.) J.J. Sm. & Epiphyte \\
\hline 95 & Dendrobium subulatum (B1.) Lindl. & Epiphyte \\
\hline 96 & Dendrobium uniflorum Griff. & Epiphyte \\
\hline 97 & Dendrochilum pallidiflavens B1. & Epiphyte \\
\hline 98 & Dimorphorchis lowii (Lindl.) Rolfe & Epiphyte \\
\hline 99 & Dimorphorchis rossii Fowlie & Epiphyte \\
\hline 100 & Dipodium ensifolium F. Muell. & Epiphyte \\
\hline 101 & Dipodium pictum (Lindl.) Rchb. f. & Epiphyte \\
\hline 102 & Dipodium scandens (B1.) J.J. Sm. & Epiphyte \\
\hline 103 & Doritis pulcherrima Lindl. & Epiphyte \\
\hline 104 & $\begin{array}{l}\text { Dyakia hendersoniana } \\
\text { (Rchb. f.) Christenson }\end{array}$ & Epiphyte \\
\hline 105 & Eria biglandulosa J.J. Sm. & Epiphyte \\
\hline 106 & Eria bractescens Lindl. & Epiphyte \\
\hline 107 & Eria braddonii Rolfe. & Epiphyte \\
\hline 108 & Eria citrina Ridl. & Epiphyte \\
\hline 109 & Eria javanica (Sw.) Bl. & Epiphyte \\
\hline 110 & Eria jenseniana J.J. Sm. & Epiphyte \\
\hline 111 & Eria leiophylla Lindl. & Epiphyte \\
\hline 112 & Eria longissima Rchb. f. & Epiphyte \\
\hline 113 & Eria ornata (B1.) Lindl. & Epiphyte \\
\hline 114 & Eria pannea Lindl. & Epiphyte \\
\hline 115 & Eria pulchella Lindl. & Epiphyte \\
\hline 116 & Eria quadricolor J.J. Sm. & Epiphyte \\
\hline 117 & Eria xanthocheila Ridl. & Epiphyte \\
\hline 118 & Eulophia graminea Lindl. & Terrestrial \\
\hline 119 & Eulophia spectabilis (Dennst.) Suresh & Terrestrial \\
\hline 120 & Flickingeria aurieloba (J.J. Sm.) J.J. Wood. & Epiphyte \\
\hline 121 & Flickingeria bicostata (J.J. Sm.) Hawkes & Epiphyte \\
\hline 122 & Flickingeria fimbriata (B1.) Hawkes. & Epiphyte \\
\hline 123 & Flickingeria xantholeuca (Rchb. f.) Hawkes. & Epiphyte \\
\hline 124 & Gastrochilus patinatus (Ridl.) Schltr. & Epiphyte \\
\hline 125 & Grammatophyllum speciosum $\mathrm{Bl}$. & Epiphyte \\
\hline 126 & $\begin{array}{l}\text { Grammatophyllum stapeliiflorum } \\
\text { (T. \& B.) J.J. Sm. }\end{array}$ & Epiphyte \\
\hline 127 & Grosourdya appendiculata (B1.) Rchb. f. & Epiphyte \\
\hline 128 & Grosourdya muscosa (Rolfe) Garay. & Epiphyte \\
\hline 129 & Liparis compressa (B1.) Lindl. & Terrestrial \\
\hline 130 & Liparis condylobulbon Rchb. $\mathrm{f}$. & Terrestrial \\
\hline 131 & Liparis elegans Lindl. & Terrestrial \\
\hline 132 & Liparis lacerata Ridl. & Terrestrial \\
\hline 133 & Ludisia discolor (Ker-Gawl.) A. Rich. & Terrestrial \\
\hline 134 & Luisia curtisii Seidenfaden & Epiphyte \\
\hline 135 & Macodes petola (B1.) Lindl. & Terrestrial \\
\hline 136 & Malaxis latifolia (Lindl.) Garay. & Terrestrial \\
\hline 137 & Micropera fuscolutea (Lindl.) Garay & Epiphyte \\
\hline 138 & Micropera callosa Garay. & Epiphyte \\
\hline 139 & Nephelaphyllum pulchrum B1. & Terrestrial \\
\hline 140 & Nervilia discolor (B1.) Schltr. & Terrestrial \\
\hline 141 & Nervilia uniflora (F. Muell.) Schltr. & Terrestrial \\
\hline
\end{tabular}


Table 1. (Continued) List of orchid species found in West Borneo, Indonesia.

\begin{tabular}{|c|c|c|}
\hline No. & Orchid name & $\begin{array}{l}\text { Growth } \\
\text { classification }\end{array}$ \\
\hline 142 & Oberonia ciliolata Hook.f. & Epiphyte \\
\hline 143 & Oberonia patentifolia Ames \& C. Schwenf. & Epiphyte \\
\hline 144 & Paphiopedilum hookerae Rchb.f. & Terrestrial \\
\hline 145 & Paphiopedilum kolopakingii Fowlie & Terrestrial \\
\hline 146 & Paphiopedilum lowii (Lind.) Stein. & Terrestrial \\
\hline 147 & Papilionanthe hookeriana (Rchb. f.) Schltr. & Terrestrial \\
\hline 148 & Paraphalaenopsis denevei (J.J. Sm.) Hawkes & Epiphyte \\
\hline 149 & $\begin{array}{l}\text { Paraphalaenopsis serpentilingua } \\
\text { (J.J. Sm.) A.D. Hawkes }\end{array}$ & Epiphyte \\
\hline 150 & Phalaenopsis amabilis (Lindl.) Bl. & Epiphyte \\
\hline 151 & Phalaenopsis bellina (Rchb. f.) E.A. Cristenson & Epiphyte \\
\hline 152 & Phalaenopsis cornucervi (Breda) Bl. \& Rchb. f. & Epiphyte \\
\hline 153 & Phalaenopsis gigantea J.J. Sm. & Epiphyte \\
\hline 154 & Phalaenopsis maculata Rchb. f. & Epiphyte \\
\hline 155 & Phalaenopsis manii Rchb. f. & Epiphyte \\
\hline 156 & Phalaenopsis modesta J.J. Sm. & Epiphyte \\
\hline 157 & Phalaenopsis pantherina $\mathrm{Rchb} . \mathrm{f}$. & Epiphyte \\
\hline 158 & Phalaenopsis sumatrana Korth. \& Rchb. f. & Epiphyte \\
\hline 159 & Pholidota imbricata Lindl. & Epiphyte \\
\hline 160 & Pholidota ventricosa (Roxb.) Lindl. & Epiphyte \\
\hline 161 & Plocoglottis acuminata Bl. & Terrestrial \\
\hline 162 & Plocoglottis lowii J.J. Sm. & Terrestrial \\
\hline 163 & Pomatocalpa kunstleri (Hk. f.) J.J. Sm. & Epiphyte \\
\hline 164 & Pomatocalpa latifolia J.J. Sm. & Epiphyte \\
\hline 165 & Pomatocalpa naevata J.J. Sm. & Epiphyte \\
\hline 166 & Pomatocalpa spicata Breda & Epiphyte \\
\hline 167 & Porphyroglottis maxwelliae Ridl. & Epiphyte \\
\hline 168 & $\begin{array}{l}\text { Pteroceras cladostachyum } \\
\text { (Hk. f.) H. Ae. Peders }\end{array}$ & Epiphyte \\
\hline 169 & Pteroceras pallidum (B1.) Holtt. & Epiphyte \\
\hline 170 & Renanthera elongata (Hk. f.) Lindl. & Epiphyte \\
\hline 171 & Robiquetia spathulata (B1.) J.J.Sm. & Epiphyte \\
\hline 172 & Schoenorchis micranta Reinw. & Epiphyte \\
\hline 173 & Schoenorchis secundiflora (Ridl.) J.J. Sm. & Epiphyte \\
\hline 174 & Spathoglottis plicata $\mathrm{Bl}$. & Terrestrial \\
\hline 175 & Taeniophyllum obtusum B1. & Epiphyte \\
\hline 176 & Tainia paucifolia J.J. Sm. & Terrestrial \\
\hline 177 & Thecopus secunda (Ridl.) Seidenf. & Epiphyte \\
\hline 178 & Thecostele alata (Roxb.) Par.\& Reichb. f. & Epiphyte \\
\hline 179 & Thelasis carinata $\mathrm{B} 1$. & Epiphyte \\
\hline 180 & Thelasis micrantha (Brogn.) J.J. Sm. & Epiphyte \\
\hline 181 & Thelasis obtusa $\mathrm{Bl}$. & Epiphyte \\
\hline 182 & Thrixspermum acuminatissimum Rchb. f. & Epiphyte \\
\hline 183 & Thrixspermum amplexicaule (B1.) Rchb. f. & $\begin{array}{l}\text { Epiphyte } \\
\text { and terrestrial }\end{array}$ \\
\hline 184 & Thrixspermum centipeda Lour. & Epiphyte \\
\hline 185 & Trichoglottis bipenicillata J.J. Sm. & Epiphyte \\
\hline 186 & Trichoglottis cirrhifera Teijsm \& Binn. & Epiphyte \\
\hline 187 & Trichoglottis geminata (T. \& B.) J.J. Sm. & Epiphyte \\
\hline 188 & Trichoglottis retusa $\mathrm{Bl}$. & Epiphyte \\
\hline 189 & Trichoglottis smithii J.J. Sm. & Epiphyte \\
\hline 190 & Trichoglottis uexkulliana J.J. Sm. & Epiphyte \\
\hline 191 & Trichotosia aporina (Hk. f.) Krzl. & Epiphyte \\
\hline 192 & Trichotosia ferox B1. & Epiphyte \\
\hline 193 & Trichotosia gracilis Lindl. & Epiphyte \\
\hline 194 & Vanda scandens Holttum & Epiphyte \\
\hline 195 & Vanda dearei Rchb. f. & Epiphyte \\
\hline 196 & Vanilla diabolica O’Byrne. & Epiphyte \\
\hline 197 & Vanilla griffithii Rchb. f. & Epiphyte \\
\hline
\end{tabular}

Some species with high economic value such as Arachnis breviscapa, Arachnis hookeriana, Bulbophyllum beccarii, Bulbophyllum dearei, Coelogyne pandurata, Cymbidium bicolor, Dendrobium hallieri, Dendrobium singkawangense, Dimorphorchis lowii, Vanda dearei, Paphiopedilum hookerae, Paphiopedilum kolopakingii, and Paphiopedilum lowii have become endangered. Among these orchids, Paphiopedilum lowii still can be found in Sanggau and Sintang counties. Bulbophyllum beccarii exact microhabitat, which supports its specific associated mycorrhiza.

Noteworthy taxa include two species from genus Paphiopedilum (Paphiopedilum hookerae and Paphiopedilum kolopakingii), two species from the genus Paraphalaenopsis (Paraphalaenopsis denevei and Paraphalaenopsis serpentilingua), Cymbidium bicolor, Dimorphorchis rossii, and several species from genus Phalaenopsis, none of which are common in nature because they are highly prized by poachers or they have vanished as their habitat is destroyed. These orchids are hardly found in their habitats but are more easily found outside their habitats. They are found in Serawak (Malaysia), in nurseries in Java, and some big cities in Indonesia as well as some parts of the Western hemisphere. Especially, Paraphalaenopsis serpentilingua is now critically endangered, and it can only be found in Sintang county. However, the Bogor Botanical Garden in Indonesia has successfully cultivated it. Likewise, Macodes petola, Anoectochilus albolineatus, and Ludisia discolor have become extremely rare and critically endangered. Only a few of these plants were encountered. These orchids live in shady, moist, and humically rich habitats. Moreover, Phalaenopsis gigantea, Paraphalaenopsis denevei, and Phalaenopsis amabilis are also critically endangered. They have never been found in their habitats and are hardly found in the markets. The author recommends that all vulnerable, endangered, and critically endangered species should be cultivated before they become extinct. Local government intervention and participation in conservation, cultivation as well as marketing of orchids are necessary so that parties will not directly take the plants from their habitat.

\section{Literature Cited}

Banks, D.P. 1999. Tropical orchids of Indonesia. Periplus Edition (HK) Ltd., Singapore.

Banks, D.P. 2004a. Handy pocket guide to the orchids of Indonesia. Periplus Edition (HK) Ltd., Singapore.

Banks, D.P. 2004b. Orchids: Cultivation, propagation and varieties. Murdoch Books Pty Ltd., Sydney, Australia.

Chan, C.L., A. Lamb, P.S. Shim, and J.J. Wood. 1994. Orchids of Borneo Vol. 1. The Sabah Society Kota Kinabalu in association with The Royal Botanic Gardens Kew, Surrey, UK.

Clayton, D. 2002. The genus Coelogyne a synopsis. Natural History Publications (Borneo) in association with The Royal Botanic Gardens Kew, Surrey, UK.

Cribb, P. 1997. Slipper orchids of Borneo. Natural History Publications, Kota Kinabalu, Malaysia.

Dixon, K.W., P.K. Shepagh, L.B. Russell, and J.C. Philip. 2003. Orchid conservation. Natural History Publications (Borneo), Kota Kinabalu, Malaysia.

Dressler, R.L. 1993. Phylogeny and classification of the orchid family. Dioscorides Press, Portland, OR.

Global Forest Watch. 2002. Indonesia: Overview. 25 Sept. 2002. 28 Apr. 2006. <http://www. globalforestwatch.org/English/Indonesia/index. htm>.

Indonesian Ministry of the Environment. 1990. Undang-Undang No. 5 Tahun 1990 Tentang: 
Konservasi Sumberdaya Alam Hayati dan Ekosistemnya. 28 Apr. 2006. <http://www. menlh.go.id/i/art/pdf_1038295823.pdf>.

Indonesian Ministry of the Environment. 1994. Undang-Undang No. 5 Tahun 1994 Tentang: Pengesahan United Nations Convention on Biological Diversity. <http://www.menlh.go.id/ i/art/pdf_1038297639.pdf $>$.

Indonesian Ministry of the Environment. 1999. Peraturan Pemerintah No. 7 Tahun 1999 Tentang: Pengawetan Jenis Tumbuhan dan Satwa. 28 Apr. 2006. <http://www.menlh.go.id/i/art/pdf 1038451276.pdf $>$.
Irawati. 2002. Pelestarian jenis anggrek di Indonesia. Proseding Seminar Anggrek Nasional, Yogyakarta.

Marinelli, J. 2004. Plant. Dorling Kindersley Limited, London, UK.

Moeso, S. 1988. Mengenal anggrek alam Indonesia. Penebar Swadaya, Jakarta.

O'Byrne, P. 2001. A to Z of South East Asia orchid species. Orchid Society of South East Asia, Singapore.

Teo, C.K.H. 1995. Native orchids of Peninsular Malaysia. Times Book International, Singapore.
Vermeulen, J.J. 1991. Orchids of Borneo Vol. 2. Bulbophyllum. Bentham-Moxon Trust, Royal Botanic Gardens Kew, Surrey, UK.

Wood, J.J. 1997. Orchids of Borneo Vol. 3. The Sabah Society Kota Kinabalu in association with The Royal Botanic Gardens Kew, Surrey, UK.

Wood, J.J. 2001. Dendrochilum of Borneo. Natural History Publications (Borneo), Kota Kinabalu in association with The Royal Botanic Gardens Kew, Surrey, UK.

Wood, J.J. 2003. Orchids of Borneo Vol. 4. The Sabah Society Kota Kinabalu in association with Royal Botanic Gardens Kew, Surrey, UK. 